\title{
Ablación láser de recubrimientos de politetrafluoretileno (PTFE) aplicados sobre sustratos EN AW-5251
}

\author{
Guillermo R. Guerrero Vaca ${ }^{\mathrm{a}, \bowtie}$, Lorenzo Sevilla Hurtado ${ }^{\mathrm{b}}$, Carlos Soriano Reyes \\ ${ }^{a}$ Universidad de Córdoba, Departamento de Mecánica, ctra. Madrid-Cádiz km 396, CP 14071, Córdoba, España

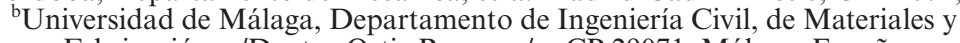 \\ Fabricación, c/Doctor Ortiz Ramos s/n, CP 29071, Málaga, España \\ 'IK4-Tekniker, Unidad de Tecnologías Avanzadas de Fabricación, c/Iñaki Goenaga 5, CP 20600, Eibar, España \\ Autor para la correspondencia: guillermo.guerrero@uco.es
}

Enviado: 17 Febrero 2014; Aceptado: 16 Marzo 2014; Publicado on-line: 28 O ctubre 2014

RESUMEN: En la actualidad se utilizan diversos sistemas de eliminación de recubrimientos ricos en politetrafluoretileno (PTFE) sobre sustratos metálicos: granallado, pirólisis, procedimientos químicos y combinaciones de ellos, presentando todos ellos serias dificultades. En el presente trabajo se propone el uso de ablación láser para eliminar recubrimientos ricos en PTFE que previamente han sido aplicados en chapas de la aleación de aluminio magnesio EN AW-5251 H34. Para ello se han analizado los valores del límite elástico convencional, resistencia a la tracción, alargamiento porcentual, energía retenida por impacto y dureza. También se ha evaluado la distribución del tamaño de grano a nivel microestructural, el tamaño medio de grano ASTM y la distribución de partículas constituyentes. Los estudios se han efectuado en tres etapas sucesivas de aplicación y eliminación láser del recubrimiento. De forma paralela, se determinan y se comparan las propiedades con los mismos sustratos y etapas, pero eliminados por pirólisis. De la comparación de los resultados se concluye que la eliminación por ablación láser no produce un empobrecimiento añadido de las propiedades de los sustratos y que puede convertirse en una alternativa industrial a los procedimientos de eliminación tradicionales.

PALABRAS CLAVE: Ablación láser; Eliminación recubrimiento; Eliminación teflón; Recubrimiento PTFE

Citation / Cómo citar este artículo: Guerrero Vaca, G.R., Sevilla Hurtado, L., Soriano Reyes, C. (2014) "Ablación láser de recubrimientos de politetrafluoretileno (PTFE) aplicados sobre sustratos EN AW-5251". Rev. Metal. 50(4): e027. doi: http://dx.doi.org/10.3989/revmetalm.027.

ABSTRACT: Laser ablation of polytetrafluoroethylene (PTFE) coatings applied on EN AW-5251 substrates. Currently the most common used methods for removing PTFE rich coatings on metal substrates are: grinding, pyrolysis, chemical processes or a combination of these. While effective, all present serious difficulties. In this paper the use of laser ablation to remove PTFE rich coatings, which have previously been applied to sheets of aluminum magnesium alloy EN AW-5251 H34, is proposed. For this purpose the values of the yield strength, tensile strength, percent elongation, impact energy retained and hardness are analyzed. Equally, the grain size distribution at the microstructural level, the ASTM average grain size and distribution of constituent particles have been evaluated. Measurements were performed to three successive stages of application and laser coating removal. Moreover, the previous set of properties have been determined for the same substrates and stages but using pyrolysis to remove the coating. Comparison of the results shows that the removal by laser ablation does not cause any reduction in the properties of the substrates and may become an industrial alternative to traditional disposal procedures.

KEYWORDS: Coating removal; Laser ablation; PTFE coating; Teflon removal

Copyright: (C) 2014 CSIC. This is an open-access article distributed under the terms of the Creative Commons Attribution-Non Commercial (by-nc) Spain 3.0 License. 


\section{INTRODUCCIÓN}

Los recubrimientos de politetrafluoretileno (PTFE) son desarrollados por corporaciones industriales como Du Pont de Nemours \& Co, Whitford Company, Daikin, Grebe Group, entre otros, para ser aplicados sobre sustratos metálicos, cerámicos, vidrios, a modo de recubrimiento multicapa. Estas resinas pueden ser aplicadas con formulaciones líquidas, en polvo e incluso una combinación de ambas formas. Habitualmente las capas finales polimerizan mediante acción térmica entre los 300 a $450{ }^{\circ} \mathrm{C}$, pudiendo alcanzar espesores de 10 a $80 \mu \mathrm{m}$ de forma habitual.

Estos recubrimientos son extensamente utilizados, desde hace más de cuatro décadas, debido a sus excelentes propiedades de antiadherencia, ya que son hidrofóbicos y oleofóbicos en proporción elevada, presentando una alta capacidad de desmoldeo, alta inercia química, baja fricción, resistencia térmica elevada y un buen comportamiento protector contra la corrosión. Por otra parte, algunas formulaciones permiten el contacto con alimentos. El precio de estas resinas, sin embargo, es elevado.

Las aplicaciones antiadherentes con fluoropolímeros particularmente con PTFE, son comunes en sustratos metálicos de aluminio, sobre todo cuando exista contacto alimentario, y se deba exigir bajo peso y buenas propiedades mecánicas al sustrato. Esto ocurre, entre otros casos, con las aleaciones de Al-Mg de la serie 5000.

Conviene considerar, por otra parte, que los recubrimientos ricos en PTFE, como cualquier recubrimiento, poseen una determinada vida útil, ya que se desgastan, se rayan, se degradan y se ensucian, por lo que deben ser extraídos del sustrato periódicamente para ser sustituidos por nuevos recubrimientos fluoropoliméricos (Drobny, 2006).

Los procesos de eliminación que actualmente se aplican en la industria van desde los procedimientos de granallado o chorreado, con empleo de una gran variedad de abrasivos, hasta la pirólisis, pasando por el uso de decapantes químicos o, incluso, una combinación de ellos (Mckeen, 2006). Sin embargo, estos procedimientos no están exentos de dificultades. Así, en el caso del granallado se debe tener especial precaución debido al riesgo de incendio o deflagración si el polvo producido en la operación de granallado se mezcla con las partículas de fluoropolímero y se expone a una llama o, simplemente, a temperaturas superiores a $424{ }^{\circ} \mathrm{C}$. Este procedimiento resulta poco eficiente dado que los recubrimientos suelen quedar fuertemente anclados al sustrato y poseen una dureza superficial elevada.

El procedimiento pirolítico resulta mucho más eficiente, siendo el más utilizado, aun cuando resulte poco recomendable debido a los niveles de emisión de contaminantes que se producen. La eliminación por pirólisis de los fluoropolímeros debe realizarse con elevada precaución, en ambientes muy ventilados y con sistemas de protección personal. Los gases de la carbonización son tóxicos y deben ser tratados antes de emitirse a la atmósfera, dado que la inhalación de los vapores liberados en la pirólisis pueden producir una enfermedad conocida como gripe del teflón ${ }^{\circledR}$ o fiebre por humos de polímeros (Drobny, 2001).

Los sistemas de eliminación por procedimientos químicos resultan, de igual modo, poco eficientes debido a la inercia química de estos recubrimientos.

Frente a las anteriores, la eliminación de revestimientos como pinturas, barnices u óxidos, e incluso restos biológicos o restos de petróleo (Mateo et al., 2005; López et al., 2010) utilizando un haz láser, mediante el procedimiento conocido como ablación láser, es un proceso que tiene importantes ventajas en comparación con las técnicas convencionales de eliminación por medio de abrasivos, químicos o pirolítico (Flores et al., 1998; Duarte y Peças, 1998; Schmidt et al., 1999; Schmidt et al., 2000; Schmidt et al., 2003; Mongelli et al., 2005; Shuöcker y Bielak, 2007; Coutouly et al., 2009). Entre ellas, cabe destacar la posibilidad de eliminar la capa superficial sin dañar el sustrato o minimizando el daño, la supresión de productos químicos y la producción mínima de gases y vapores contaminantes (Freiwald et al., 1998; Marimuthu et al., 2010). Nuevos desarrollos proponen la combinación con sistemas de chorro de agua para eliminar los restos, si es que aparecen, del proceso ablativo (Madhukhar et al., 2013). Los estudios realizados sobre sustratos de aluminio se han centrado fundamentalmente en las aleaciones EN AW-2024 T3 y EN AW-7075 T6, con distintas tipologías de fuentes láser, sobre recubrimientos de resinas epoxi o resinas de epoxi-poliamida y capa final de poliuretano, de aplicación en el sector aeronáutico (Head y Niedzielski, 1991; Pantelakis y Haidemenopoulos, 1998, Klingenberg et al., 2007).

Sin embargo, no se conoce suficientemente si alguna de las ventajas citadas en la eliminación por ablación láser de otros recubrimientos puede tener el mismo nivel de éxito para los recubrimientos en aplicaciones antiadherentes con resinas ricas con PTFE. De igual modo, tampoco se conoce si la acción de un haz láser apropiado para la extracción del recubrimiento puede producir una transformación o daño en las propiedades mecánicas de sustratos de aluminio.

Identificar un procedimiento de eliminación adecuado mediante el proceso de ablación láser y determinar la influencia sobre el sustrato metálico puede resultar un avance tecnológico de alto interés para las industrias especializadas en las aplicaciones antiadherentes de resinas fluoropoliméricas.

En este trabajo se estudia el proceso de eliminación por ablación láser de un tipo de recubrimiento fluoropolimérico rico en PTFE aplicado sobre substratos de la aleación de aluminio EN AW-5251 H34. 
A su vez, se determinan, comparan y analizan las características microestructurales y propiedades mecánicas de los sustratos de aluminio tratados, utilizando en la comparación la misma aleación de aluminio y tipo de recubrimiento eliminado mediante pirólisis.

\section{MATERIALES Y MÉTODOS}

\subsection{Condiciones generales}

En este trabajo, sobre el material del sustrato, se han determinado las propiedades mecánicas de límite elástico convencional, carga de rotura a tracción, alargamiento porcentual, energía retenida al impacto y dureza Vickers. También se ha caracterizado la microestructura, realizándose un seguimiento de las posibles modificaciones microestructurales (tamaño de grano ASTM y distribución de la población de partículas constituyentes) en las distintas etapas de los procesos. El sustrato está constituido por chapas de la aleación de aluminio EN AW-5251 H34, que fueron recubiertas con un fluoropolímero, rico en PTFE, de uso común en aplicaciones antiadherentes del sector de alimentación.

Los procedimientos de aplicación de los recubrimientos ricos en PTFE, los procedimientos de eliminación del recubrimiento y el programa de ensayos a los que son sometidos los sustratos han sido propuestos de acuerdo a Guerrero (2013).

Se prepararon 20 chapas de $140 \times 120 \mathrm{~mm} \mathrm{y}$ $1,2 \mathrm{~mm}$ de espesor, mediante el cizallado de chapas comerciales de EN AW-5251 H34, aleación de aluminio no tratable térmicamente que permite el contacto alimentario, con elevada deformabilidad, adecuada dureza y alta resistencia a corrosión. Esta aleación se emplea en la fabricación de recipientes, soportes, bandejas para trasvasar, hornear y manipular masas para alimentación, así como soporte o sustrato de aplicaciones antiadherentes. Se crearon dos grupos de 10 chapas, el primero destinado a la eliminación por ablación láser y el segundo a la eliminación por pirólisis. Las probetas procesadas se ensayaron después de cada ciclo de aplicación y eliminación, reiterándose 3 veces cada ciclo. El ciclo contempla las etapas siguientes: (i) granallado de los sustratos, (ii) aplicación de los recubrimientos, (iii) polimerizado de los recubrimientos, (iv) eliminación de los recubrimientos por ablación láser o bien por pirólisis, (v) granallado de la superficie tras la eliminación de los recubrimientos y (vi) fabricación de las probetas.

Junto a los datos suministrados por el proveedor sobre la composición química de la aleación de aluminio utilizada, los resultados obtenidos por microanálisis de rayos $\mathrm{X}(\mathrm{EDS})$ mediante un microscopio electrónico de barrido, recogidos en la Tabla 1, confirmaron los valores especificados en la norma UNE 38347 para la aleación EN AW-5251.
TABla 1. Composición química ( $\%$ en peso) de las chapas EN AW-5251 ensayadas

\begin{tabular}{cccccccc}
\hline $\mathbf{M g}$ & $\mathbf{S i}$ & $\mathbf{F e}$ & $\mathbf{M n}$ & $\mathbf{C u}$ & $\mathbf{Z n}$ & $\mathbf{C r}$ & $\mathbf{T i}$ \\
\hline 1,9 & 0,15 & 0,4 & 0,5 & 0,1 & 0,3 & 0,02 & 0,01 \\
\hline
\end{tabular}

Para la caracterización microestructural se prepararon muestras metalográficas, utilizando en su montaje resina acrílica de curado en frío, pulidas hasta sílice coloidal de $1 / 4 \mu \mathrm{m}$ y anodizadas electrolíticamente con reactivo Barker. Las observaciones se efectuaron con microscopio óptico bajo luz polarizada. Las mediciones de los parámetros relacionadas con los tamaños de grano y de partículas constituyentes se realizaron mediante software de metalografía cuantitativa por análisis de imagen. La estructura de la aleación del sustrato en su estado de recepción H34 (endurecido por deformación en frío y estabilizado con un grado final de dureza $1 / 2$ duro, es decir, que la resistencia a tracción es aproximadamente un valor intermedio entre el estado recocido O y el estado más duro producido H38) se muestra en la Figura 1.

Asimismo, en la Figura 2 se muestran la morfología y distribución de la población de partículas constituyentes (intermetálicos) sobre la matriz $\alpha$ (solución sólida rica en $\mathrm{Al}$ ).

Previamente a la aplicación del recubrimiento con PTFE, las probetas sufrieron un pretratamiento de preparación superficial mediante chorreado con corindón blanco, de granulometría P120 según FEPA (Federation of the European Producers of Abrasives). Este pretratamiento es recomendado por el fabricante de las resinas, con objeto de asegurar un buen anclaje al sustrato del recubrimiento.

Los fluoropolímeros utilizados en los recubrimientos fueron formulaciones de Whitford Corporation, clasificadas como recubrimientos antiadherentes para contacto alimentario multicapa de base acuosa. La aplicación de estos recubrimientos se suele realizar sobre elementos que deban poseer cualidades de antiadherencia en su superficie. Estos recubrimientos pueden actuar desde la temperatura ambiente hasta $200-220^{\circ} \mathrm{C}$.

Siguiendo las especificaciones del fabricante se aplicó, en primer lugar, la formulación líquida de Marlite X-Treme 135.500 Z Blue Prime, por pulverización mediante una pistola aerográfica tipo HVLP (alto volumen-baja presión) de forma manual. Inmediatamente se realizó un secado a $150{ }^{\circ} \mathrm{C}$ en una estufa industrial, durante 5 minutos. La capa seca de imprimación alcanzó un espesor de $8 \mu \mathrm{m}$. Posteriormente se aplicó una capa intermedia de Marlite X-Treme 235.490 Z Nero Mety, por último, una capa final tras la capa intermedia, sin secado previo, es decir, húmedo sobre húmedo, de Marlite X-Treme 245.491 Nero Metalizzato Finish, también mediante pulverización con una pistola HVLP. 

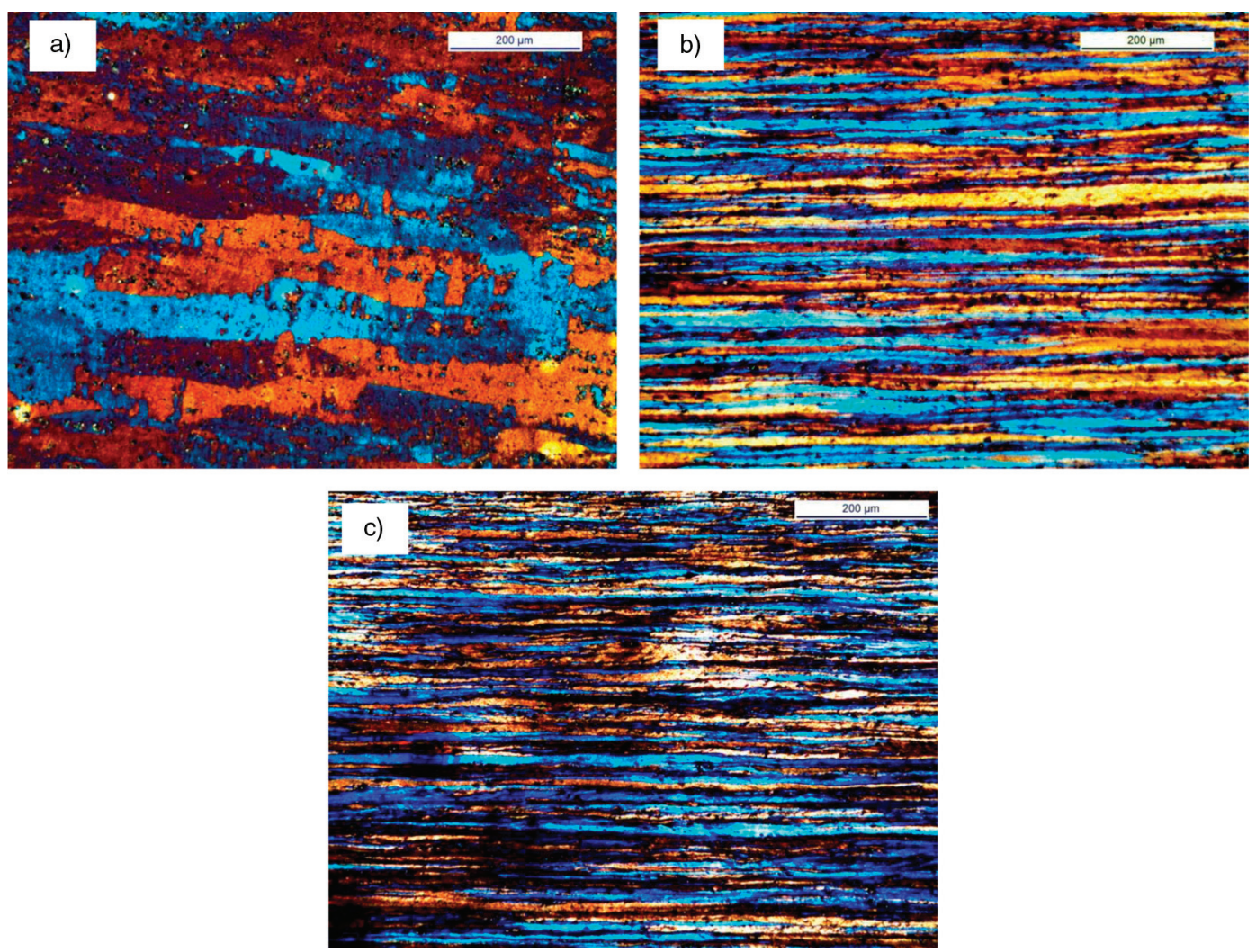

FiguRA 1. Estructura de deformación correspondiente al estado de recepción H34: (a) Estructura de grano en la superficie de la chapa de $140 \times 120 \times 1,2 \mathrm{~mm}$; (b) En la sección de un plano normal a la chapa y paralelo al lado de $120 \mathrm{~mm}$ y

(c) En la sección de un plano normal a la chapa y paralelo al lado de $140 \mathrm{~mm}$.

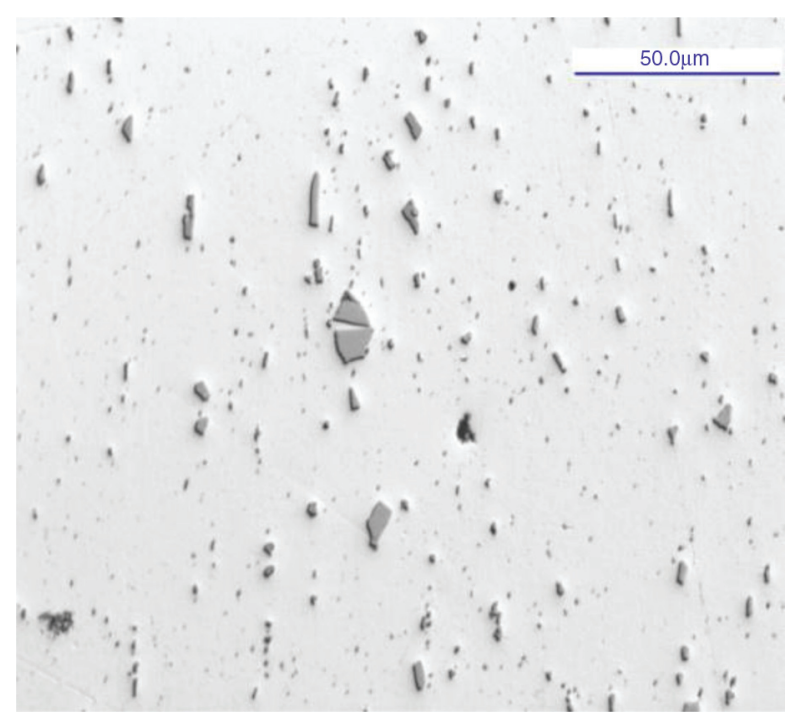

Figura 2. Micrografía en estado de suministro, pulida sin ataque.

El proceso de aplicación se finalizó con un ciclo de polimerización de la capa intermedia y final en un horno eléctrico estático, con una duración del ciclo de 45 minutos. El ciclo alcanza la temperatura de $410-415^{\circ} \mathrm{C}$ en 20 minutos, se mantiene en este nivel durante 7 minutos y con posterioridad se enfría hasta temperatura ambiente en 18 minutos.

\subsection{Características de los recubrimientos}

La caracterización del recubrimiento se realizó mediante la medición de la microdureza con un ultra-microdurómetro con indentador Vickers, aplicándose el método de Oliver y Pharr (1992). Los ensayos se han realizado con una carga de $10 \mathrm{mN}$, obteniéndose valores de $70 \mathrm{MPa}$, para una profundidad de $0,3 \mu \mathrm{m}$, y $60 \mathrm{MPa}$, a $2 \mu \mathrm{m}$. El espesor final del recubrimiento se midió según los criterios de la norma UNE-EN ISO 2808 resultando un espesor $e=19,09 \mu \mathrm{m}$, con una varianza $\sigma=4,09 \mu \mathrm{m}$. El color del recubrimiento final en el producto acabado se ha asociado a un RAL 9004. Se realizó un ensayo de mojabilidad de los recubrimientos con el agua, obteniéndose un ángulo de contacto superficial estático de $112^{\circ}$, un ángulo de retroceso de $103^{\circ}$ y un ángulo de avance de $114^{\circ}$, valores todos ellos compatibles con este tipo de recubrimientos (McKeen, 2006).

Finalmente, con el fin de conocer la fracción de radiación láser incidente reflejada por la superficie 
del recubrimiento en función de la longitud de onda de emisión del láser, se caracterizó el espectro de reflectancia del recubrimiento mediante un espectrofotómetro. Se determinó que en un láser de Nd:YAG, con longitud de onda de emisión de $1064 \mathrm{~nm}$, el valor de la reflectancia, sobre este recubrimiento, estaba dentro del intervalo 5-6\%.

\subsection{Sistemas de eliminación de los recubrimientos}

Cuando se realiza la eliminación de recubrimientos a nivel industrial con fuentes láser, es usual utilizar equipos de media a alta potencia (Barletta et al., 2006; Prinsloo et al., 2007). Las últimas tendencias en el ámbito de la aeronáutica se extienden hacia equipos robotizados con fuentes láser de potencias que alcanzan hasta $6 \mathrm{~kW}$ en láseres con emisión en el espectro infrarrojo (Arthur et al., 2008). Los láseres de Nd:YAG y $\mathrm{CO}_{2}$ son los sistemas más recomendados para la extracción de fluoropolímeros (Riva y Pezzetti, 1997; Ol'khov et al., 2010). También se conocen sistemas de eliminación de películas delgadas de PTFE con láseres de excímero $\left(\mathrm{F}_{2}\right)$ con una longitud de onda de $157 \mathrm{~nm}$ (George et al., 2009; Reznicêkova et al., 2011). El proceso es conocido como ablación láser y puede ser efectuado mediante láseres pulsados o continuos. En el caso de láseres pulsados, y cuando el flujo de radiación es muy intenso, el láser vaporiza el recubrimiento y se crea un plasma, el cual genera una onda de choque que produce la rotura y eliminación del recubrimiento (Miller y Haglund, 1998). En cambio, cuando se utilizan láseres de onda continua, el mecanismo de ablación es fundamentalmente por descomposición térmica.

En el caso que se estudia se ha empleado una fuente láser con un generador de Nd:YAG de onda continua $(\mathrm{CW})$, bombeado por diodos y con emisión en el espectro infrarrojo cercano $(\lambda=1064 \mathrm{~nm})$, con una potencia máxima de $2200 \mathrm{~W}$. El haz láser ha sido guiado a la zona de proceso a través de una fibra óptica de sección circular de 0,6 mm de diámetro. Posteriormente, tras atravesar una lente de colimación de $160 \mathrm{~mm}$ de distancia focal y una lente de prefocalización de $900 \mathrm{~mm}$, el haz es introducido en un escáner de espejos galvanométricos sujeto a un eje lineal $\mathrm{Z}$ de una máquina de control numérico. Finalmente, el haz es enfocado sobre el área de interés mediante una lente convergente de $200 \mathrm{~mm}$ de focal, generando una huella circular de $1 \mathrm{~mm}$ de diámetro, en un campo de trabajo de $120 \times 120 \mathrm{~mm}$. Las probetas se han dispuesto sobre una garra de vacío sujeta a un eje lineal $\mathrm{X}$.

Para llevar a cabo la ablación, tras una parametrización previa, se ha seleccionado un conjunto de parámetros que permiten una eliminación completa, eficiente y sin daño de la superficie del sustrato (Tabla 2). De esta forma se ha programado un barrido lineal de la huella láser de $120 \mathrm{~mm}$ de longitud, oscilando a una frecuencia de $22 \mathrm{~Hz}$.
TABla 2. Parámetros utilizados en la ablación láser del recubrimiento de PTFE sobre aleaciones de EN AW-5251

\begin{tabular}{lcccc}
\hline $\begin{array}{l}\text { Potencia } \\
\text { (W) }\end{array}$ & $\begin{array}{c}\text { Frecuencia de } \\
\text { barrido (Hz) }\end{array}$ & $\begin{array}{c}\text { Longitud de } \\
\text { barrido (mm) }\end{array}$ & $\begin{array}{c}\text { Avance } \\
\left(\mathbf{m m ~ m i n}^{-1}\right)\end{array}$ & $\begin{array}{c}\mathbf{N}^{\circ} \\
\text { pasadas }\end{array}$ \\
\hline 600 & 22 & 120 & 500 & 1 \\
\hline
\end{tabular}

La velocidad de desplazamiento del eje lineal $\mathrm{X}$ de coordenadas se ha fijado en $500 \mathrm{~mm} \mathrm{~min}^{-1}$.

La otra tecnología de eliminación de recubrimientos utilizada ha sido la extracción pirolítica. En este caso las piezas recubiertas han sido introducidas en un horno continuo de $12 \mathrm{~m}$ de longitud equipado con resistencias eléctricas. La potencia nominal del horno es de $60 \mathrm{~kW}$. La velocidad de avance de la cinta ha sido de $0,27 \mathrm{~m} \mathrm{~min}^{-1}$ en un ciclo de 45 minutos de duración. El ciclo de despolimerizado por pirólisis garantiza la carbonización de los recubrimientos al alcanzar temperaturas de $500{ }^{\circ} \mathrm{C}$ durante un intervalo de tiempo de 10 minutos.

En el caso de la eliminación por ablación láser, las condiciones de parámetros seleccionadas permiten una tasa de extracción de $600 \mathrm{~cm}^{2} \mathrm{~min}^{-1}$, con una densidad de energía de $50 \mathrm{~J} \mathrm{~cm}^{-2}$.

Para la eliminación mediante el procedimiento pirolítico se ha obtenido una tasa de extracción de $1600 \mathrm{~cm}^{2} \mathrm{~min}^{-1} \mathrm{y}$ un valor de fluencia $140 \mathrm{~J} \mathrm{~cm}^{-2}$.

\subsection{Análisis de la microestructura recristalizada (O)}

Inicialmente se establecieron las condiciones de recristalización de la estructura de deformación correspondiente al estado de suministro, comprobándose que dicha recristalización se produce a $395^{\circ} \mathrm{C}$, siguiendo la rampa de calentamiento correspondiente a la primera aplicación del PTFE. La Figura 3 muestra la estructura de grano en la condición de material recristalizado.

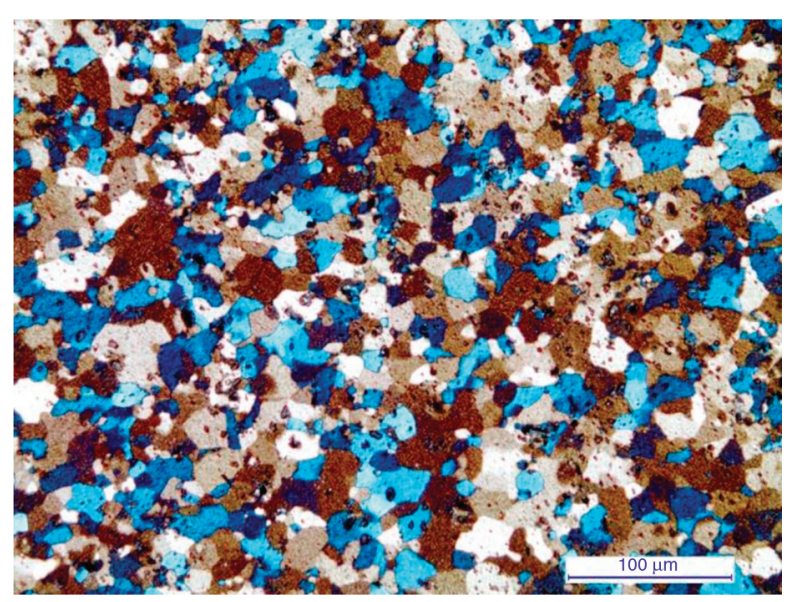

FIgURA 3. Micrografía obtenida en EN AW-5251 a la temperatura de $395^{\circ} \mathrm{C}$, según la curva de polimerización del recubrimiento de PTFE (estructura recristalizada). 
Para la medición del tamaño de grano ASTM se aplicó el método planimétrico de intercepción, definido en la norma ASTM E-112. Los valores obtenidos se muestran en la Tabla 3.

Con objeto de determinar el valor relativo de los distintos tamaños de grano perceptibles en la micrografía, se empleó la ecuación (1) que propone la norma ASTM E-112, siendo $G$ el tamaño de grano (adimensional) y $l$ la longitud media interceptada, en milímetros.

$$
G=-6,664 \times \log _{10}(l)-3,288
$$

Cuando se introduce en la ecuación (1) el valor medio de la longitud interceptada de todos los tamaños de grano, se puede asociar un tamaño medio de grano ASTM a toda la estructura, obteniéndose un valor de 8,34 (8,5 según valor más próximo por indicación de la norma), para la condición de la estructura recristalizada a $395{ }^{\circ} \mathrm{C}$.

La relativa heterogeneidad en los tamaños de los granos recristalizados viene inducida por los mecanismos de recristalización. Éstos pueden clasificarse en mecanismos continuos (se distribuyen homogéneamente por toda la estructura) y en discontinuos (se distribuyen heterogéneamente). En las aleaciones de base aluminio, la recristalización está fuertemente influenciada por la presencia de partículas constituyentes y dispersoides. Debido al desajuste cristalino causado por su presencia, gruesas partículas intermetálicas (1-10 $\mu \mathrm{m})$ están rodeadas por regiones deformadas de alta energía almacenada, que proporcionan localmente una mayor fuerza motriz para la recristalización (Humphreys y Hatherl, 1995). Las partículas gruesas ricas en $\mathrm{Fe} / \mathrm{Mn}$ (diámetro $>1-2 \mu \mathrm{m}$ ) crean una deformación incompatible en la matriz circundante durante la deformación en frío. Durante la recristalización, nuevos granos pueden nuclearse en las zonas deformadas alrededor de las partículas. El mecanismo de recristalización se conoce como PSN (Particle Stimulated Nucleation), y se inicia de una manera discontinua en lugares de alta energía almacenada, tales como en las proximidades de partículas gruesas.

En relación a las partículas constituyentes, se identificaron dos tipos, tanto en el estado de suministro (H34) como en la condición de material recristalizado $(\mathrm{O})$. Mediante espectroscopía de energía dispersiva (EDS), estas partículas fueron identificadas como ricas en $\mathrm{Al}(\mathrm{Fe}, \mathrm{Mn}) \mathrm{Si}$ y $\mathrm{Mg}_{\mathrm{x}} \mathrm{Si}$, respectivamente.

\subsection{Obtención de las propiedades mecánicas}

Para la determinación del límite elástico convencional $\left(R_{p 0,2}\right)$, resistencia a la tracción $\left(R_{m}\right)$ y alargamiento de rotura $(A \%)$ se utilizó una prensa electromecánica de $100 \mathrm{kN}$. Los ensayos se realizaron conforme a lo establecido en UNE-EN ISO 6892-1. Las dimensiones de las probetas se tomaron según el Anexo B de la citada norma.

Para la determinación de la energía retenida por el impacto se utilizó un péndulo Charpy de $300 \mathrm{~J}$ de energía nominal, con percutor de $2 \mathrm{~mm}$ de diámetro. Se mecanizaron probetas de sección reducida con entalla en forma de "U". Los ensayos correspondientes se realizaron conforme a lo dispuesto en la norma UNE-EN ISO 148-1.

La dureza Vickers se midió utilizando un durómetro universal, con carga de ensayo de $10 \mathrm{kp}(98,07 \mathrm{~N})$. Los correspondientes ensayos se realizaron según UNE-ISO 6507-1.

Las propiedades mecánicas, correspondientes al estado de suministro se midieron y están recogidas en la Tabla 4.

\section{RESULTADOS}

\subsection{Características microestructurales}

Se realizó un estudio de la estructura de grano en cada uno de los tres ciclos contemplados en el experimento y por cada una de las vías de eliminación. Las variaciones de las micrografías son pequeñas con respecto a la micrografía obtenida en la estructura recristalizada, no obstante, se muestran las micrografías obtenidas tras el tercer ciclo de aplicación por cada una de las vías de eliminación propuestas (Figs. 4 y 5).

También es posible asociar un tamaño medio de grano ASTM a toda la microestructura y, de este modo, se ha determinado este valor en cada una de las etapas relevantes del proceso. Estos datos se recogen en la Tabla 5.

Similar análisis se realizó sobre la población de partículas constituyentes (intermetálicos), determinándose el valor relativo de las áreas de las partículas sobre la superficie. Los resultados se muestran en la Tabla 6.

\subsection{Propiedades mecánicas}

Los resultados de los ensayos mecánicos efectuados sobre las diversas probetas con objeto de estudiar las variaciones se muestran en las Figuras 6,

TABLA 3. Intervalo de valores de la longitud interceptada de grano ${ }^{\text {a }}$ de EN AW-5251 tras el ciclo de polimerizado de un recubrimiento de PTFE hasta $395^{\circ} \mathrm{C}$

\begin{tabular}{lccccc}
\hline Valor mínimo $(\boldsymbol{\mu m})$ & Valor máximo $(\boldsymbol{\mu m})$ & Valor medio $(\boldsymbol{\mu m})$ & Desviación estándar $(\boldsymbol{\mu m})$ & Suma $(\mu \mathrm{m})$ & $\mathbf{N}^{\circ}$ de medidas \\
\hline 5,98 & 33,12 & 17,78 & 5,56 & 2187 & 123 \\
\hline
\end{tabular}

${ }^{a}$ Método planimétrico ASTM E-112. 
TABlA 4. Propiedades mecánicas en estado de suministro de EN AW-5251 H34

\begin{tabular}{|c|c|c|c|c|c|}
\hline $\begin{array}{l}\begin{array}{l}\text { Estado de } \\
\text { suministro }\end{array} \\
\end{array}$ & $\begin{array}{c}\text { Límite elástico } \\
\boldsymbol{R}_{p 0,2}(\mathrm{MPa}) \\
\end{array}$ & $\begin{array}{c}\text { Resistencia a tracción } \\
\boldsymbol{R}_{m}(\mathrm{MPa}) \\
\end{array}$ & $\begin{array}{c}\text { Alargamiento } \\
A(\%)\end{array}$ & $\begin{array}{c}\text { Dureza Vickers } \\
(\text { HV 10) }\end{array}$ & $\begin{array}{l}\text { Energía retenida por } \\
\text { sección } R\left(\mathrm{~J} \mathrm{~mm}^{-2}\right)\end{array}$ \\
\hline H34 & 216 & 256 & 9,4 & 82 & 0,385 \\
\hline
\end{tabular}

${ }^{a}$ Dureza medida sobre el plano D-T.

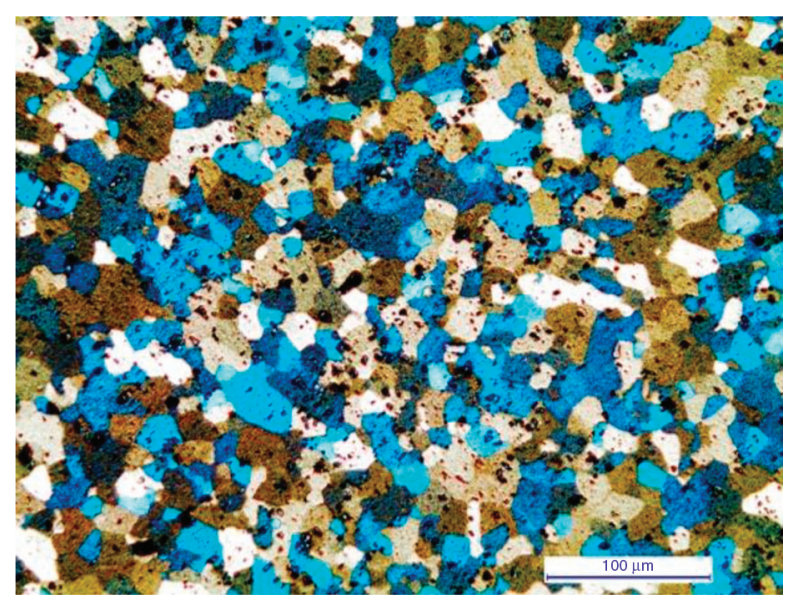

Figura 4. Micrografía que muestra la estructura de grano tras el tercer ciclo completo de aplicación y eliminación del PTFE por ablación láser.

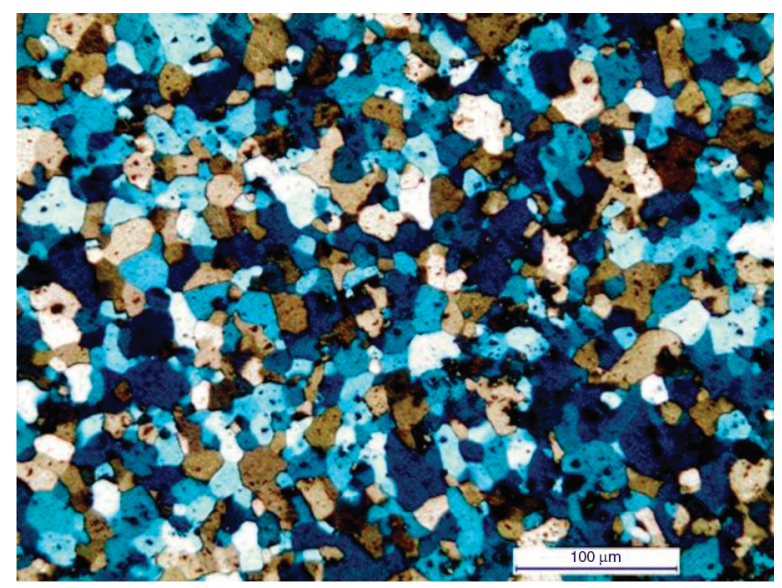

Figura 5. Micrografía que muestra la estructura de grano tras el tercer ciclo completo de aplicación y extracción del PTFE por pirólisis.

7 y 8 , en las que se estudian las variaciones de la resistencia a tracción, el alargamiento porcentual a la rotura y la dureza Vickers. En ellas se reflejan los valores medios obtenidos en series de tres ensayos efectuados para cada propiedad mecánica, en cada una de las fases y para cada método de eliminación del recubrimiento de las chapas de EN AW-5251.

Otras propiedades mecánicas también han sido estudiadas, como son: límite elástico convencional y energía retenida al impacto cuyos resultados se muestran en las Tablas 7 y 8 .

\section{DISCUSIÓN}

El equipo láser empleado ha permitido la extracción por ablación láser del recubrimiento rico en PTFE mediante un mecanismo de descomposición térmica. Las tasas de remoción obtenidas han sido relativamente elevadas $\left(600 \mathrm{~cm}^{2} \mathrm{~min}^{-1}\right)$, comparadas con otros procesos y medios similares (Barletta et al., 2006; Klingenberg et al., 2007; Wolf et al., 2009), mostrando que el sistema láser seleccionado ha sido eficiente. Es previsible que otros sistemas láser como los de $\mathrm{CO}_{2}$ (con longitudes de onda cercanas al espectro de absorción máximo del fluoropolímero), así como el uso de fuentes láser pulsadas (con duraciones de pulso del orden de los nanosegundos y alta energía por pulso), arrojarían tasas de extracción aún más elevadas, cercanas e incluso superiores a las tasas obtenidas por pirólisis. Los equipos en la vanguardia están incorporando sistemas de reconocimiento mediante escaneo superficial para adaptar de forma automática los parámetros de eliminación (velocidad, potencia, intensidad, pulso) a las posibles variaciones del recubrimiento (color, brillo, espesor), pudiéndose aplicar igualmente a la eliminación de recubrimientos ricos en fluoropolímeros.

Los valores de tasas de eliminación de los procedimientos pirolíticos son sustancialmente superiores $\left(1300 \mathrm{~cm}^{2} \mathrm{~min}^{-1}\right)$, si bien deben tenerse en cuenta las limitaciones, anteriormente expuestas, que presenta este sistema.

Las propiedades mecánicas de los sustratos de aluminio estudiados sufren una intensa modificación, con valores similares, tanto en los procedimientos por pirólisis como en los realizados por láser. Estas modificaciones resultan apreciables ya desde la primera etapa y aparecen tras la aplicación del recubrimiento rico en PTFE. La aleación recristaliza por completo y pasa del estado $\mathrm{H} 34$ al estado $\mathrm{O}$.

La modificación estructural produce: (i) un aumento del alargamiento porcentual desde el $9,4 \%$ a niveles de $20,3-21,2 \%$; (ii) un aumento de la energía absorbida $R$ desde $0,385 \mathrm{~J} \mathrm{~mm}^{-2}$ a $0,498-0,522 \mathrm{~J} \mathrm{~mm}^{-2} \mathrm{y}$, consecuentemente, un aumento de la tenacidad; (iii) una disminución del límite elástico $R_{p 0,2}$ desde $216,3 \mathrm{MPa}$ a 81,8-92,4 MPa; (iv) una disminución de la resistencia a tracción $R_{m}$ desde 255,8 MPa a 195,3-203,5 MPa, siendo en todo caso la propiedad mecánica menos afectada; (v) una disminución de la dureza Vickers desde 82,3 HV10 a 50,3-51,7 HV10. 
TABLA 5. Valor del tamaño de grano medio ASTM en el estado EN AW-5251 O y tras cada unos de los tres ciclos completos de aplicación de recubrimiento de PTFE y eliminación por ablación láser y por pirolisis

\begin{tabular}{|c|c|c|c|c|c|c|}
\hline $\begin{array}{l}\text { Estado } \\
\text { recristalizado }(\mathrm{O})\end{array}$ & $\begin{array}{l}\text { Ablación } \\
\text { láser (1ª) }\end{array}$ & $\begin{array}{l}\text { Ablación } \\
\text { láser }\left(2^{\mathrm{a}}\right)\end{array}$ & $\begin{array}{l}\text { Ablación } \\
\text { láser (3 })\end{array}$ & $\begin{array}{l}\text { Eliminación } \\
\text { Pirólisis }\left(1^{\text {a }}\right)\end{array}$ & $\begin{array}{l}\text { Eliminación } \\
\text { Pirólisis }\left(2^{\mathrm{a}}\right)\end{array}$ & $\begin{array}{l}\text { Eliminación } \\
\text { Pirólisis }\left(3^{\mathbf{a}}\right)\end{array}$ \\
\hline 8,34 & 8,27 & 8,14 & 8,11 & 8,13 & 8,09 & 8,03 \\
\hline
\end{tabular}

TABLA 6. Valor (\%) del área formada por partículas constituyentes en el estado EN AW-5251 O y tras cada unos de los tres ciclos completos de aplicación de recubrimiento de PTFE y eliminación por ablación láser y por pirolisis

\begin{tabular}{|c|c|c|c|c|c|c|}
\hline $\begin{array}{l}\text { Estado } \\
\text { recristalizado (O) } \\
\end{array}$ & $\begin{array}{l}\text { Ablación } \\
\text { láser }\left(1^{\mathbf{a}}\right)\end{array}$ & $\begin{array}{l}\text { Ablación } \\
\text { láser }\left(2^{\mathrm{a}}\right) \\
\end{array}$ & $\begin{array}{l}\text { Ablación } \\
\left.\text { láser ( } 3^{\mathbf{a}}\right)\end{array}$ & $\begin{array}{l}\text { Eliminación } \\
\text { Pirólisis }\left(\mathbf{1}^{\mathbf{a}}\right)\end{array}$ & $\begin{array}{l}\text { Eliminación } \\
\text { Pirólisis }\left(2^{\mathrm{a}}\right)\end{array}$ & $\begin{array}{l}\text { Eliminación } \\
\text { Pirólisis }\left(3^{\mathrm{a}}\right)\end{array}$ \\
\hline 3,18 & 3,06 & 3,12 & 3,21 & 3,20 & 3,14 & 3,16 \\
\hline
\end{tabular}

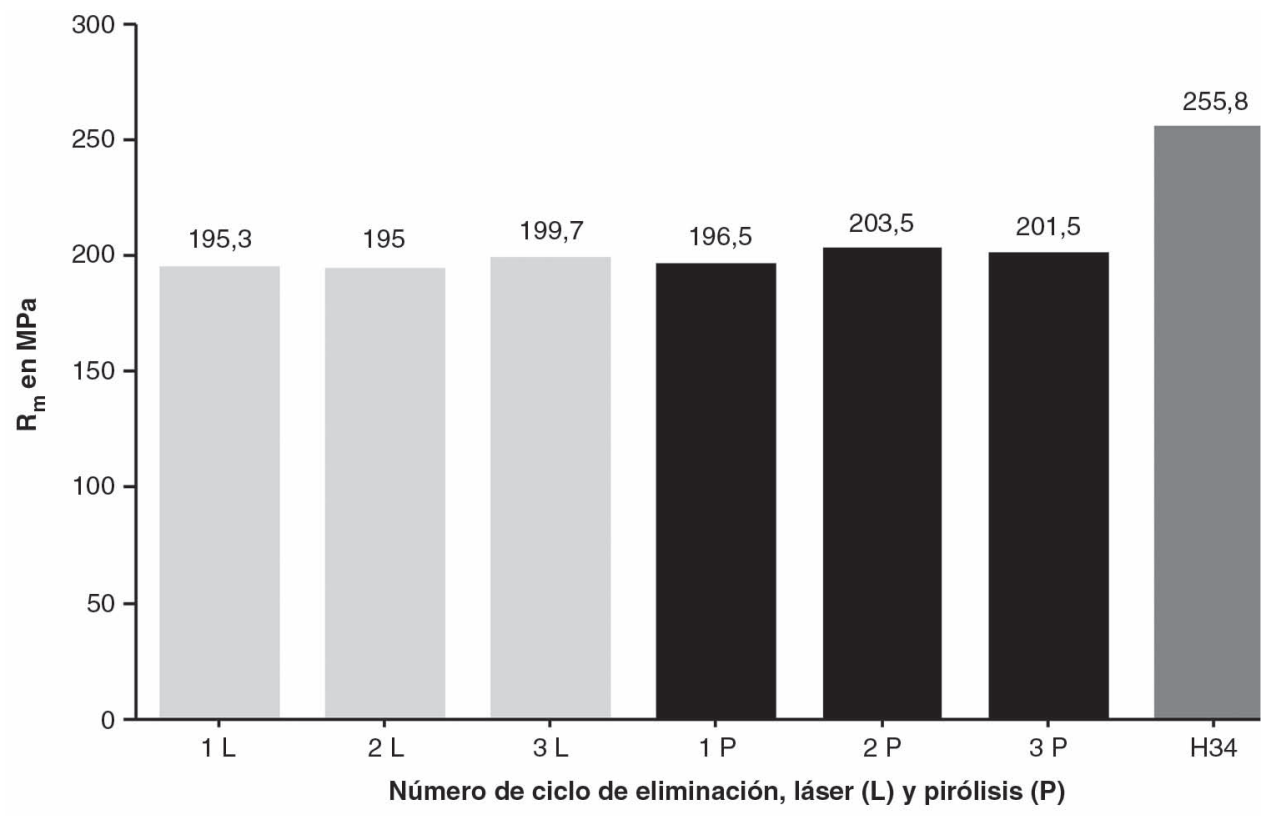

FIgURA 6. Valor de la resistencia a tracción $R_{m}$ de EN AW-5251, en el estado H34 y tras cada uno de los tres ciclos completos de aplicación de recubrimiento de PTFE y eliminación por ablación láser (L) y por pirólisis (P).

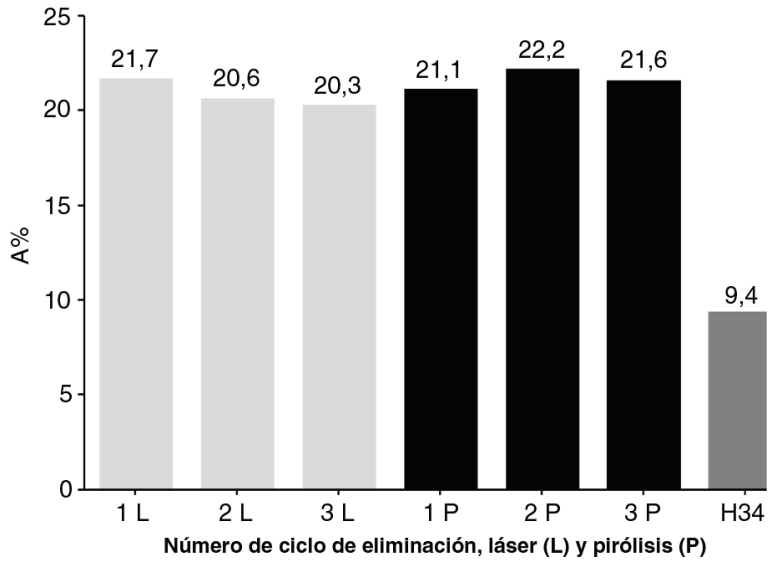

FIGURA 7. Valor del alargamiento porcentual de rotura $A(\%)$ de EN AW-5251, en el estado H34, y tras cada uno de los tres ciclos completos de aplicación de recubrimiento de PTFE y eliminación por ablación láser $(\mathrm{L})$ y por pirólisis $(\mathrm{P})$.

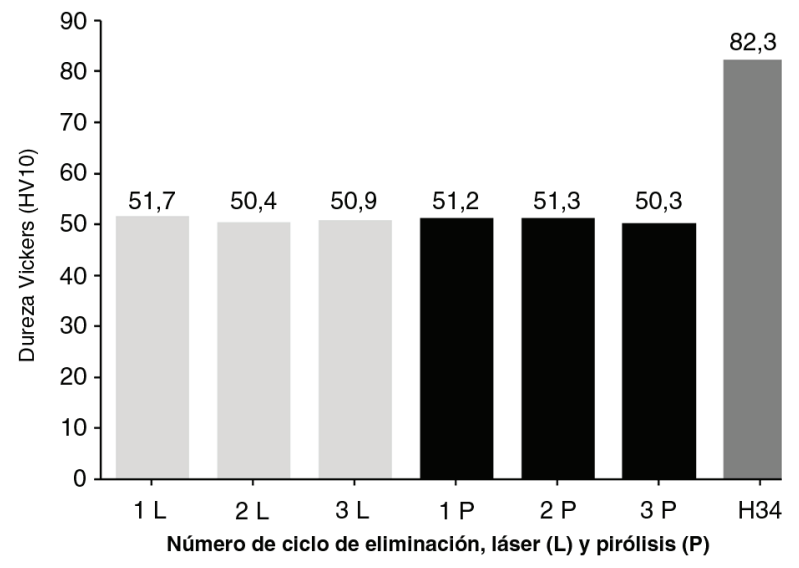

FIGURA 8. Valor de la dureza Vickers (HV10) de EN AW-5251, en el estado de suministro (H34) y después de cada uno de los tres ciclos completos de aplicación de recubrimiento de PTFE y eliminación por ablación láser $(\mathrm{L})$ y por pirólisis $(\mathrm{P})$. 
TABLA 7. Valor del límite elástico $R_{p 0,2}(\mathrm{MPa})$ de EN AW-5251, en el estado (H34) y tras cada uno de los tres ciclos completos de aplicación de recubrimiento de PTFE y eliminación por ablación láser $(\mathrm{L})$ y por pirólisis $(\mathrm{P})$

\begin{tabular}{|c|c|c|c|c|c|c|}
\hline H34 & $\begin{array}{l}\text { Ablación } \\
\text { láser }\left(1^{\mathbf{a}}\right)\end{array}$ & $\begin{array}{l}\text { Ablación } \\
\text { láser }\left(2^{\mathrm{a}}\right)\end{array}$ & $\begin{array}{l}\text { Ablación } \\
\text { láser (3a) }\end{array}$ & $\begin{array}{l}\text { Eliminación } \\
\text { Pirólisis }\left(1^{\mathbf{a}}\right)\end{array}$ & $\begin{array}{l}\text { Eliminación } \\
\text { Pirólisis }\left(2^{\mathrm{a}}\right)\end{array}$ & $\begin{array}{l}\text { Eliminación } \\
\text { Pirólisis }\left(3^{\mathrm{a}}\right)\end{array}$ \\
\hline 216,3 & 92,4 & 86,8 & 85,4 & 81,8 & 86,1 & 85,8 \\
\hline
\end{tabular}

TABLA 8. Valor de la energía retenida por sección $R\left(\mathrm{~J} \mathrm{~mm}^{-2}\right)$ de EN AW-5251, en el estado (H34) y tras cada uno de los tres ciclos completos de aplicación de recubrimiento de PTFE y eliminación por ablación láser (L) y por pirólisis (P)

\begin{tabular}{|c|c|c|c|c|c|c|}
\hline H34 & $\begin{array}{l}\text { Ablación } \\
\left.\text { láser (1 }{ }^{\mathbf{a}}\right)\end{array}$ & $\begin{array}{l}\text { Ablación } \\
\text { láser }\left(2^{2}\right)\end{array}$ & $\begin{array}{l}\text { Ablación } \\
\text { láser }\left(3^{a}\right)\end{array}$ & $\begin{array}{l}\text { Eliminación } \\
\text { Pirólisis }\left(1^{\text {a }}\right)\end{array}$ & $\begin{array}{l}\text { Eliminación } \\
\text { Pirólisis }\left(2^{\mathrm{a}}\right)\end{array}$ & $\begin{array}{l}\text { Eliminación } \\
\text { Pirólisis }\left(3^{a}\right)\end{array}$ \\
\hline 0,385 & 0,504 & 0,504 & 0,500 & 0,522 & 0,502 & 0,498 \\
\hline
\end{tabular}

Respecto a las características microestructurales observadas, se ha determinado que existe una variación pequeña del tamaño de grano ASTM desde 8,03 a 8,39 , correspondiendo en todos los casos a un tamaño 8 u 8,5 según la norma ASTM. La variación del porcentaje de partículas constituyentes sobre la matriz de aluminio oscila entre el 2,94 al 3,21. Ambos resultados indican que la población de partículas constituyentes y el tamaño de grano quedan muy levemente afectados, pudiendo atribuirse dichas variaciones a la propia variación del proceso. Esto puede explicarse debido al hecho de que la mayor parte de la población de partículas está constituida por precipitados primarios ricos en $\mathrm{Fe}$ y $\mathrm{Mn}$ y que, debido al alto punto de fusión de éstos (mayor de $700{ }^{\circ} \mathrm{C}$ ), no pueden disolverse $\mathrm{y}$ posteriormente precipitar a las temperaturas empleadas.

En cuanto a las propiedades mecánicas, se ha podido observar que las variaciones que se producen una vez que la aleación ha recristalizado y se encuentra en el estado EN AW-5251 O, se originan con similar intensidad en ambos procedimientos y fases de eliminación del recubrimiento estudiado. De este modo se puede observar que: (i) la resistencia a tracción oscila entre $195 \mathrm{MP}$ (láser) y 203,5 MPa (pirólisis); (ii) el límite elástico varía entre 81,8 MPa (pirólisis) y 92,4 MP (láser); (iii) el alargamiento porcentual oscila entre $20,3 \%$ (láser) y $22,2 \%$ (pirólisis); (iv) la energía absorbida $R$ oscila entre $0,500 \mathrm{~J} \mathrm{~mm}^{-2}$ (láser) y $0,522 \mathrm{~J} \mathrm{~mm}^{-2}$ (pirolisis); (v) la dureza Vickers oscila entre 50,3 HV10 (pirólisis) y 51,7 HV10 (láser).

Las variaciones existentes en las propiedades mecánicas ensayadas no muestran ninguna tendencia, apuntando nuevamente hacia variaciones inherentes al propio proceso.

Por tanto, no se aprecian diferencias que supongan una mayor idoneidad entre una u otra técnica de eliminación de los recubrimientos, ni en las propiedades mecánicas ni en las características microestructurales de los sustratos del tipo EN AW-5251, tras tres ciclos de aplicación y remoción de recubrimientos del tipo PTFE.
Aunque algunos autores han relacionado fallos o mermas de las propiedades en los sustratos por aplicación de la ablación láser para la eliminación de recubrimientos, entre ellos la fragilización estructural debido a inclusiones de hidrógeno por fenómenos fotoquímicos (Pantelakis y Haidemenopoulos, 1998), o efectos por microfundición superficial o empobrecimiento añadido de las propiedades de fatiga (Klingenberg et al., 2010), ninguno de estos efectos parece haberse mostrado en el presente estudio.

Tras la ablación láser, cabe esperar un comportamiento similar en aquellas aleaciones de la serie 5000 de $\mathrm{Al}-\mathrm{Mg}$ con contenido de magnesio que no produzcan precipitación de la fase $\beta$, en el diagrama binario Al-Mg (generalmente en $\mathrm{Al}_{3} \mathrm{Mg}_{2}$ y $\mathrm{Al}_{8} \mathrm{Mg}_{5}$ ) y que se encuentren endurecidas en frío. En contraposición, en las que dispongan de precipitación de la fase $\beta$ es previsible que también disminuyan sus propiedades mecánicas, si bien, probablemente, en menor intensidad.

\section{CONCLUSIONES}

El conjunto de resultados reunidos en el presente estudio permite llegar a las siguientes conclusiones:

Las propiedades mecánicas y características microestructurales de la aleación de aluminio EN AW-5251 H34, tras los ciclos de polimerizado del recubrimiento de PTFE y posterior eliminación por pirólisis o por ablación láser, sufren una importante modificación, si bien en proporciones muy similares e independientemente de la vía de eliminación.

La eliminación por ablación láser de recubrimientos fluoropoliméricos sobre sustratos EN AW-5251 es una técnica viable que alcanza una tasa de eliminación elevada, aunque algo inferior a la que se obtiene con la eliminación por pirólisis.

La eliminación por ablación láser minimiza los serios inconvenientes que posee la eliminación pirolítica (susceptible de producir la enfermedad conocida como gripe del teflón ${ }^{\circledR}$ o fiebre por humos de polímeros) debido a la facilidad de 
ubicar sistemas de aspiración adosados al cabezal de la fuente láser pudiendo convertirse en una alternativa industrial viable, frente a los procedimientos de eliminación convencionales de recubrimientos de PTFE.

\section{REFERENCIAS}

Arthur, J., Bowman, R., Straw, R. (2008). Robotic laser coating removal system. Final Report, Environmental Security Technology Certification Program, Air Force Research Laboratory, Ohio, USA.

ASTM E-112 (2010). Standard test methods for determining average grain size. ASTM International, USA.

Barletta, M., Gisario, A., Taglaferri, V. (2006). Advance in paint stripping from aluminium substrates. J. Mater Process. Tech. 173 (2), 232-239. http://dx.doi.org/10.1016/j. jmatprotec.2005.11.029.

Coutouly, J.F., Deprez, P., Breaban, F., Longuemard, J.P. (2009). Optimisation of a paint coating ablation process by $\mathrm{CO}_{2}$ TEA laser: Thermal field modelling and real-time monitoring of the process. J. Mater. Process. Tech. 209 (17), 5730 5735. http://dx.doi.org/10.1016/j.jmatprotec.2009.06.001.

Drobny, J. (2001). Effects of heat, radiation, and environment on fluoropolymers, technology of fluoropolymers. CRC Press, Boca Ratón, Florida, USA, pp. 171-182.

Drobny, J. (2006). Fluorplastic. Rapra Technology Limited, Shropshire, Reino Unido.

Duarte, J.P., Peças, P. (1998). Limpieza con láser de excímeros de papéis e pergaminos com lama. Rev. Metal. 34, 101-102. http://dx.doi.org/10.3989/revmetalm.1998.v34.i2.669.

Flores, T., Ponce, L., Moreno, B., Arronte, M., Fernándaz, M.,García, C. (1998). Nd:YAG laser in art works restoration. Rev. Metal. 34, 98-100. http://dx.doi.org/10.3989/ revmetalm.1998.v34.i2.668.

Freiwald, D., Peebles, H., Case, R. (1998). Industrial laser-based coatings removal systems. Proc. SPIE 3343, 814-820. $\mathrm{http} / / / \mathrm{dx}$. doi.org/10.1117/12.321608.

George, S.R., Leraas, J.A., Langford, S.C., Dickinson, J.T. (2009). Interaction of $157-\mathrm{nm}$ excimer laser radiation with fluorocarbon polymers. Appl. Surf. Sci. 255 (24), 9558-9561. http://dx.doi.org/10.1016/j.apsusc.2009.04.090.

Guerrero, G. (2013). Análisis comparativo de los procesos de eliminación de los recubrimientos antiadherentes fluoropoliméricos en superficies metálicas entres tecnologías láser $y$ piroliticas. Tesis Doctoral. Universidad de Málaga, Málaga, España.

Head, J.D., Niedzielski, J.P. (1991). Laser paint stripping. Wright Laboratory, Air Force Materiel Command, Ohio, USA.

Humphreys, F.J., Hatherl, M. (1995). Recrystallization and Related Annealing Phenomena. Elseiver Science, Oxford, Reino Unido.

Klingenberg, M.L., Naguy, D.A., Naguy, T.A., Straw, R.J., Joseph, C., Mongelli, G.A., Nelson, G.C., Denny, S.L., Arthur, J.J. (2007). Transitioning laser technology to support air force depot transformation needs. Surf. Coat. Tech. 202 (1), 45-57. http://dx.doi.org/10.1016/j.surfcoat. 2007.04.056.

Klingenberg, M.L., Valencia, J., Price, G., Adams, J.R., Blair, T.P. (2010). Naval application of laser ablation paint removal. Technology. Final Report, Navy Metalworking Center, USA

Lopez, A.J., Rivas, T., Lamas, J., Ramil, A., Yañez, A. (2010). Optimisation of laser removal of biological crusts in granites. Appl. Phys. A. 100 (3), 733-739. http://dx.doi. org/10.1007/s00339-010-5652-x.

Madhukhar, Y.K., Mullick, S., Nath, A.K. (2013). Development of a water-jet assisted laser paint removal process. Appl. Surf. Sci. 286, 192-205. http://dx.doi.org/10.1016/j.apsusc. 2013.09.046.

Marimuthu, S., Camara, A.M., Whitehead, D., Mativenga, P., Li, L. (2010). Laser removal of TiN coatings from WC micro-tools and in process monitoring. Opt. Laser. Technol. 42 (8), 1233-1239. http://dx.doi.org/10.1016/j. optlastec.2010.03.016
Mateo, M.P., Nicolas, G., Piñon, V., Ramil, A., Yañez, A. (2005). Laser cleaning: an alterenative method for removing oilspill fuel residues. Appl. Surf. Sci. 247 (1-4), 333-339. http://dx.doi.org/10.1016/j.apsusc.2005.01.086.

McKeen, L.W. (2006). Florinated coatings and finishes handbook. William Andrews, New York, USA.

Miller, J.C., Haglund, R.F. (1998). Laser ablation and desorption. Academic Press, San Diego, USA.

Mongelli, G., Marqusee, J., Pellerin, C. (2005). Portable handheld laser small area supplemental coatings removal system. Final Report, Headquarters Air Force Materiel Command Depot Modernization and Logistics, Ohio, USA.

Oliver, W.C., Pharr, G.M. (1992). An improved technique for determining hardness and elastic modulus using load and displacement sensing indentation experiments. $J$. Mater. Res. 7 (6), 1564-1583. http://dx.doi.org/10.1557/ JMR.1992.1564.

Ol'khov, Y.A., Allayarov, S.R., Tolstopyatov, E.M., Grakovich, P.N., Kalinin, L.A., Dobrovoll'skii, Y.A., Dixon, D.A. (2010). The effect of continuous $\mathrm{CO}_{2}$ laser radiation on the thermal and molecular-topological properties of polytetrafluoroethylene. High. Energy Chem. 44 (1), 63-74. http://dx.doi.org/10.1134/S0018143910010108.

Pantelakis, Sp.G., Haidemenopoulos, G.N. (1998). Effect of novel paint removal processes on the fatigue behavior of aluminum alloy 2024. Surf. Coat. Tech. 106 (2-3), 198-204. http://dx.doi.org/10.1016/S0257-8972(98)00526-X

Pantelakis, Sp.G., Kermanidis, Th.B., Haidemenopoulus, G.N. (1996). Mechanical behavior of $2024 \mathrm{Al}$ alloy specimen subjected to paint stripping by laser radiation and plasma etching. Theor. Appl. Fract. Mec. 25 (2), 139-146. http:// dx.doi.org/10.1016/0167-8442(96)00016-X.

Prinsloo, F.J., Van Heerden, S.P., Ronander, E. (2007). Efficient TEA $\mathrm{CO}_{2}$-laser-based coating removal system. Proc. SPIE 6346, 63462Q1-63426Q8. http://dx.doi.org/10.1117/ 12.739109 .

Řezničková, A., Chaloupka, A., Heitz, J., Kolská, J., Švorčik, V. (2011). Surface properties of polymers treated with $F_{2}$ laser. Surf. Interface Anal. 44 (3), 296-300. http://dx.doi. org/10.1002/sia.3801

Riva, D.A., Pezzetti, F. (1997). Process for removing fluorocarbon resin-based coatings. Patent US5679202.

Schmidt, M., Li, L., Spencer, J., Key, P. (1999). A comparative study of the effects of laser wavelength on laser removal of chlorinated rubber. Appl. Surf. Sci. 138-139, 418-423. $\mathrm{http}: / / \mathrm{dx}$.doi.org/10.1016/S0169-4332(98)00579-0.

Schmidt, M., Li, L., Spencer, J., Key, P. (2000). Ablation of a chlorinated rubber polymer and $\mathrm{TiO}_{2}$ ceramic mixture with a Nd:YAG laser. Appl. Surf. Sci. 154-155, 53-59. http:// dx.doi.org/10.1016/S0169-4332(99)00374-8.

Schmidt, M., Li, L., Spencer, J. (2003). An investigation into the feasibility and characteristics of using a $2.5 \mathrm{~kW}$ high power diode laser for paint stripping. J. Mater. Process. Tech. 138 (1-3), 109-115. http://dx.doi.org/10.1016/ S0924-0136(03)00057-8.

Schuöcker, G.D., Bielak, R. (2007). Laser ablation and competitive technologies in paint stripping of heavy anticorrosion coatings. Proc. SPIE 6346, 34633-34633. http://dx.doi. org/10.1117/12.739114.

UNE 38347 (2004). Aluminio y aleaciones de aluminio para forja. Serie 5000. EN-AW5251, EN-AW AlMg 2 . AENOR, España.

UNE-EN ISO 2808 (2007). Pinturas y barnices. Determinación del espesor de película. AENOR, España.

UNE-EN ISO 6892-1 (2005). Materiales metálicos. Ensayos de tracción. Parte 1 Ensayos a temperatura ambiente. AENOR, España.

UNE-EN ISO 148-1 (2009). Materiales metálicos. Ensayo de flexión por choque con péndulo Charpy. Parte 1: Método de ensayo. AENOR, España.

UNE-EN ISO 6507-1 (2006). Materiales metálicos. Ensayo de dureza Vickers. Parte 1: Método de ensayo. AENOR, España.

Wolf, K., Krincher, R., Ermalovich, J. (2009). Laser strip: a portable hand-held laser stripping device for reducing VOC, toxic and particulate emissions, Institute for Research and Technical Assistance, I.R.T.A., USA. 